\title{
Managing Discontinuity in Academic Medical Centers: Strategies for a Safe and Effective Resident Sign-Out
}

\author{
Arpana R. Vidyarthi, MD ${ }^{1}$ \\ Vineet Arora, MD, $\mathrm{MA}^{2}$ \\ Jeffrey L. Schnipper, MD, MPH \\ Susan D. Wall, $\mathrm{MD}^{4}$ \\ Robert M. Wachter, MD ${ }^{1}$ \\ ${ }^{1}$ Department of Medicine, University of California, \\ San Francisco \\ ${ }^{2}$ Department of Medicine, University of Chicago \\ ${ }^{3}$ Brigham and Women's/Faulkner Hospitalist Pro- \\ gram, Division of General Medicine, Brigham and \\ Women's Hospital and Harvard Medical School \\ ${ }^{4}$ School of Medicine, University of California, San \\ Francisco
}

\begin{abstract}
BACKGROUND: Restrictions in the hours residents can be on duty have resulted in increased sign-outs, that is, transfer of patient care information and responsibility from one physician to a cross-coverage physician, leading to discontinuity in patient care. This sign-out process, which occurs primarily in the inpatient setting, traditionally has been informal, unstructured, and idiosyncratic. Although studies show that discontinuity may be harmful to patients, this is little data to assist residency programs in redesigning systems to improve sign-out and manage the discontinuity.

PURPOSE: This article reviews the relevant medical literature, current practices in non-health professions in managing discontinuity, and summarizes the existing practice and experiences at 3 academic internal medicine hospitalist-based programs.

CONCLUSIONS: We provide recommendations and strategies for best practices to design safe and effective sign-out systems for residents that may also be useful to hospitalists working in academic and community settings. Journal of Hospital Medicine 2006;1:257-266. (C) 2006 Society of Hospital Medicine.
\end{abstract}

KEYWORDS: systems of care, medical education, patient safety.

M odern-day continuity of patient care in teaching hospitals, once remarkably high because of a cadre of sleep-deprived residents, is now peppered with breaks, each accompanied by the transfer of patient care responsibility from one resident to another; a process often referred to as a "handoff." Such transitions have long been a part of medical practice but have recently received increased attention because of restrictions in the duty hours of house staff. In July 2003 the Accreditation Council for Graduate Medical Education (ACGME) mandated reduced duty hours for all trainees in hopes of improving resident education and well-being and patient safety. ${ }^{1}$ In fact, some studies have shown improved resident well-being ${ }^{2}$ and fewer medical errors with reductions in duty hours, ${ }^{3,4}$ but the growing consensus about the negative consequences of resident fatigue on patient safety has been accompanied by parallel concerns about the potential for information loss with each break in the continuity of care. ${ }^{5,6}$

Although the tradeoff of increased discontinuity of care for fewer hours worked is sometimes characterized as an "unintended consequence" of duty hour regulations, it is in fact predictable and essential. As individuals work fewer hours, discontinuity must necessarily increase (assuming 24-hour coverage). ${ }^{7}$ The extent to which this occurs may vary, but the link is consistent. At the University of California, San Francisco (UCSF), for example, we found that compliance with new duty hour requirements for in- 
ternal medicine resulted in an average of 15 handoffs per patient during a 5-day hospitalization. Each individual intern was involved in more than 300 handoffs in an average month-long rotation, an increase of $40 \%$ since system changes were introduced to decrease duty hours. We found similar increases at Brigham and Women's Hospital (BWH) and the University of Chicago. Because U.S. teaching hospitals care for more than 6 million patients each year, ${ }^{8}$ the impact of these handoffs on the quality and efficiency of care is tremendous.

Discontinuity of care is currently managed by "sign-out," or the transfer of patient information from one physician to another. Recognizing the importance of information transfer at these vulnerable transition times for patients, the Joint Commission on Accreditation of Hospital Organizations (JCAHO) issued the 2006 National Patient Safety Goal 2E: "Implement a standardized approach to 'hand off' communications, including an opportunity to ask and respond to questions." ${ }^{9}$ Hospitals have little data to draw on to determine how to comply with this mandate and even less data to guide them in how to achieve its intended goals of improving communication and thus patient safety.

In an effort to better understand sign-outs and ways to improve this process for house staff on in-patient services, we reviewed data from the fields of aviation, communications, systems engineering, and human factors research, and we also searched the medical literature using key words "pass-off," "handoff," "sign-out," "duty hours," "work hours," and "discontinuity of care" and MeSH headings "Continuity of Patient Care Internship and Residency/*organization \& administration," "Personnel Staffing and Scheduling/*organization \& administration," and "Quality of Health Care." We also searched the websites of the Agency of Healthcare Quality and Research and the National Patient Safety Foundation. On the basis of these reviews, our experiences as hospitalist medical educators organizing resident sign-out efforts at the University of California, San Francisco, the University of Chicago, and Brigham and Women's Hospital, and our efforts leading national training sessions on sign-outs at the Society of General Internal Medicine (2004 and 2005), the Society of Hospital Medicine (2004), and the Association of Program Directors in Internal Medicine $(2005,2006)$, we propose a set of best practices regarding the content and process of sign-out in an effort to improve communication between residents caring for hospitalized pa- tients, assist programs in building safe and effective sign-out systems, and improve the quality of patient care.

\section{Effects of Discontinuity on Patient Safety}

Research on the effects of discontinuity of care, although limited, suggests it has a negative impact on patient safety. In a study that investigated the institution of code 405 (the regulation that reduced duty hours in New York State), researchers found that the presumed increase in discontinuity with decreased duty hours resulted in delayed test ordering and an increased number of hospital complications. ${ }^{10}$ Another study found that the number of potentially preventable adverse events doubled when patients were under the care of a physician from a "nonprimary" team (eg, the cross-covering intern). ${ }^{11}$ Studies have also linked resident discontinuity with longer length of stay, increased laboratory testing, and increased medication errors. ${ }^{12,13}$

\section{Managing Discontinuity: Sign-Out as the Means of Information Transfer}

In theory, more effective sign-out systems should mitigate the potential for patient harm, but there is little in the literature describing current effective sign-out practices or the best ways to design and implement such systems in the health care field. Examining information transfer mechanisms used in fields outside health care can assist in developing these systems.

\section{Information Transfer in Other Industries}

Although there is a paucity of data on sign-out in the medical literature, information transfer has been the subject of substantial research in other industries in which safety depends on effective communication.

Aviation, for example, created systems and processes to improve handoff communication in response to accidents linked to failures in information transfer. One example, the 1977 collision of 2 747 s on an airport runway in Tenerife, the Canary Islands, occurred after a garbled transmission from an air traffic controller to the cockpit of one of the aircraft. It was determined that a culture of adherence to a steep hierarchy prevented subordinates from questioning the captain's mistaken certainty that a runway was clear, ${ }^{14}$ an erroneous belief that was the basis for his decision to continue the aircraft on its course, resulting in its collision with the other airplane. 
Subsequently, commercial aviation designed systems that standardized and formalized the process of information transfer and improved teamwork and coordination. These interventions were developed on the basis of detailed observations of cockpit interactions, reviews of communication errors, and focus groups. ${ }^{15}$ Because of these efforts, today's pilots use standardized checklists to transfer information content, communicate at designated times in specific undistracted environments, and use standard language and read-backs to enhance understanding. ${ }^{16}$ The result has been a remarkable decrease in the risk of aviation crashes, one that most experts attribute in large part to these efforts to improve communication. ${ }^{17}$

Observation of how communication occurs in other high-risk industries has informed the arena of effective information transfer. For example, direct observation of information transfer at NASA, in nuclear power plants, and in the railway industry identified specific strategies for effective handoffs/ sign-outs such as standardizing the information transferred, ensuring information is up to date, limiting interruptions, and having a structured face-toface verbal interchange. ${ }^{18}$

Other strategies noted to be effective in diminishing errors are the use of a standardized phonetic alphabet to ensure that information is correctly heard and understood ${ }^{4}$ and having interactive verbal communication occur at a whiteboard. ${ }^{19}$

\section{Information Transfer in Health Care}

Those in the discipline of nursing have vast experience in the transfer of patient care information. The sign-out process employed by nurses includes face-to-face discussions, typed information, and, most commonly, taped verbal communication. ${ }^{20}$ Interestingly, this process has not been subject to detailed scrutiny, and there is little information in the literature about best practices in sign-out. Most articles in the literature on nursing handoffs are ethnographic descriptions of patient care responsibilities, ${ }^{21}$ on the basis of which, the authors advocate standardization of the information to be transferred, formalization of the channel used to communicate, and attention to increasing a culture of professionalism during sign-out in order to improve efficiency. ${ }^{20,22}$

There is little in the literature on transfer of care among physicians. Improvements in sign-out have been suggested as part of broad strategies, such as increased training and information technology sup- port, ${ }^{4,7,23,24}$ and specific strategies have been offered such as managing barriers to communication, including specific types of data when transferring care, ${ }^{25}$ and involving nurses and senior physicians in sign-outs. ${ }^{26}$ Specific outcomes data in this area have focused primarily on the use of computerized systems to improve information transfer. For example, the use of a computerized sign-out system at Brigham and Women's Hospital (BWH), linked to the hospital's information system to ensure up-todate information on patient demographics, medications, and laboratory values, has resulted in fewer errors, ${ }^{27}$ as have other similar systems. ${ }^{28}$ At the University of Washington, use of a similarly linked computerized sign-out system resulted in fewer patients being missed on rounds and improvement in the quality of sign-out and continuity of care according to resident self-reports. ${ }^{29}$ Unfortunately, fewer than $10 \%$ of hospitals have such integrated hospitalwide information systems to support the sign-out function. ${ }^{30}$

It has been noted that verbal communication, in concert with advances in technological communication, is important in information transfer in health care, ${ }^{18,31}$ especially in emergent or urgent conditions. ${ }^{32}$ For example, eliminating the phonedin report from the lab to the ER and replacing it with delivery by an electronic reporting system lacking verbal communication-resulted in $45 \%$ of emergent lab results going unchecked..$^{32}$ Structured verbal communication tools have been efficacious in improving information transfer outside the formal sign-out-for example, "read-backs," which reduced errors in the reporting of critical laboratory values, ${ }^{33}$ and the SBAR (situation, background, assessment, recommendation) tool (designed to frame the transfer of critical information), which improved physician and nurse patient care information transfer in the in-patient setting of the Kaiser Permanente health system. ${ }^{34}$

In focus groups and in response to formal and informal surveys, residents at our 3 sites suggested inclusion of the following information, provided in writing and orally, to improve sign-outs: up-to-date administrative information (eg, room number, primary care physician); patient's recent cognitive or cardiopulmonary status; problems the patient had already experienced and treatments previously tried, both successfully and unsuccessfully; patient's code status and discussions on level of care; test results or consultation recommendations that were likely to come back while covering the patient 


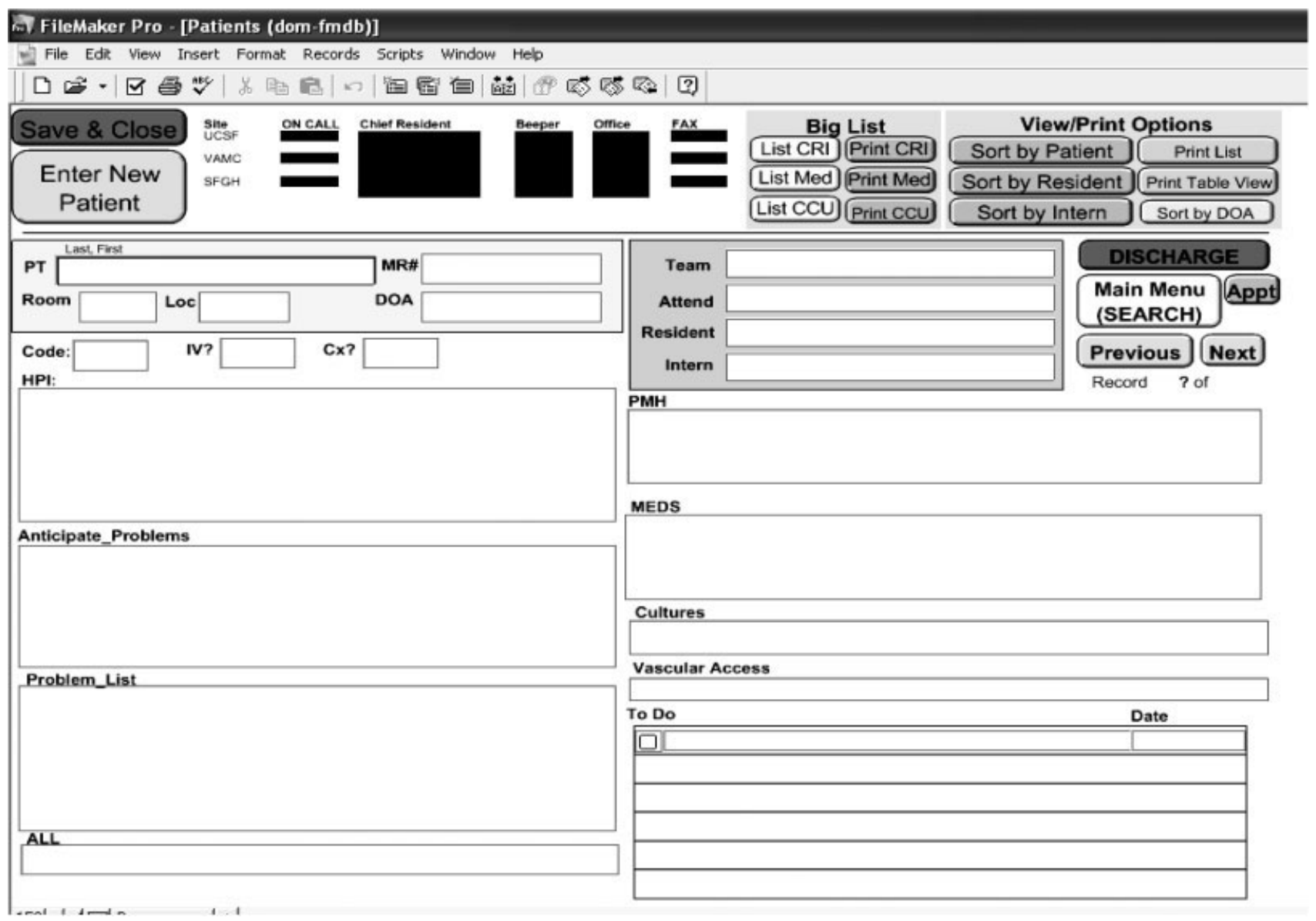

FIGURE 1. UCSF Filemaker Pro written sign-out vehicle.

and what to do with the results; and relevant psychosocial information (eg, complex family dynamics). ${ }^{35}$

\section{The Current Practice of Sign-Out}

In examining sign-outs at our 3 institutions, we found them to be unstructured and unstandardized. From discussion with faculty participating in national workshops on sign-out, we found that most sign-outs are conducted by interns, usually with little or no formal training. Templates, checklists, or other methods to standardize the content of the information transferred were rarely used.

We also noted that the vehicle for written signout is highly variable. At UCSF, different residency training programs used a variety of modalities for written sign-outs, including index cards, Excel spreadsheets, Word documents, and loose sheets of paper. Recently, the UCSF Department of Medicine designed a simple database (on Filemaker Pro ${ }^{\mathrm{TM}}$ ) that allows members of the house staff to update their sign-out information, share it with other house staff and nurses, and access it at locations throughout the hospital (Fig. 1). Although this database is not yet linked to the hospital information system (planned for 2006), anecdotally resident satisfaction with sign-out has vastly improved since its implementation. The cost of design and implementation was approximately $\$ 10,000$. At the University of Chicago, interns used Microsoft Word to create sign-out sheets containing patient summaries to transfer information. However, during structured interviews, $95 \%$ of the interns reported that these sheets were frequently lost or misplaced. ${ }^{7}$ Although medicine residents at $\mathrm{BWH}$ use a computerized system to produce sign-out sheets, this system did not guarantee complete and structured information. 
For example, a survey at BWH found that $56 \%$ of cross-covering residents said that when paged about a patient overnight, the relevant information needed to care for that patient was present less than half the time; and $27 \%$ of residents reported being paged more than 3 times in the previous 2 weeks about a test result or consultant recommendation that they did not know was pending. ${ }^{36}$

The process of sign-out also varied across disciplines and institutions. From our experiences at our sites and at the sites of faculty nationally, we found limited standardization about whether sign-out was verbal, the data transmitted, and the setting in which it was transmitted. In fact, at UCSF most residents signed out verbally "on the fly," wherever and whenever they could find the cross-coverage intern. At BWH, only $37 \%$ of residents said that sign-out occurred in a quiet place most of the time, and only $52 \%$ signed out on every patient both orally and in writing. ${ }^{36}$ At the University of Chicago, the sign-out process was characterized by outright failures in communication because of omission of needed information (ie, medications, active or anticipated medical problems, etc.) or by failureprone communication (ie, lack of face-to-face communication, illegible writing). These failures often led to uncertainty in making patient care decisions, potentially resulting in inefficient or suboptimal care. $^{35}$

\section{Strategies for Safe and Effective Sign-Out}

Given the current landscape of variability in signouts, the recognition that information lost during sign-out may result in harm to patients, and evidence of improvements in information transfer in areas outside health care, we aimed to develop mechanisms to improve the sign-out process for residents working in a hospital setting. These strategies are based on our review of the existing literature supplemented by our experiences at our 3 institutions.

\section{Content of Sign-Out}

The elements of content necessary for safe and effective sign-out can be divided into 5 broad categories (Table 1), contained in the mnemonic "ANTICipate": Administrative information, New clinical information, specific Tasks to be performed, assessment of severity of Illness, and Contingency plans or anticipated problems (Table 1, Fig. 2).

Several general points about this list should be
TABLE 1

Checklist for Elements of a Safe and Effective Written

Sign-out-ANTICipate

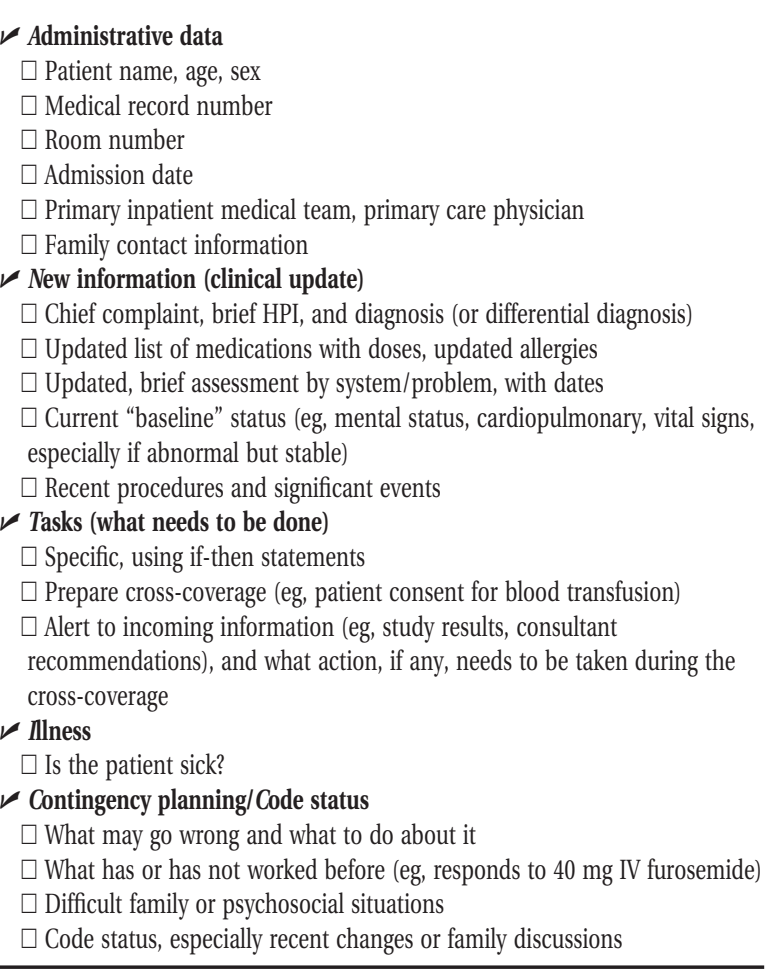

noted. First, the sign-out content is not meant to replace the chart. The information included reflects the goal of a sign-out, namely, to provide enough information to allow for a safe transition in patient care. Information we believe is not essential to the sign-out includes: a complete history and physical exam from the day of admission, a list of tasks already completed, and data necessary only to complete a discharge summary.

Sign-out must be also be a closed loop-the process of "signing in" is as important as the process of signing out. This usually entails members of the primary team obtaining information from the cross-covering physician when they resume care of the patient. The information conveyed in this case is different and includes details on events during cross-coverage such as: 1) time called to assess patient; 2) reason for call; 3) a brief assessment of the patient, including vital signs; 4) actions taken, for example, medications given and tests ordered; and 5) rationale for those actions. Some of this information may also be included in the chart as an "event note" (see Fig. 3). 
John Smith

14A-14

$123-45-67-8$

$69 \mathrm{M}$

Admitted 12/24/05

Attending: Alex Jones

Intern: Mary Kaplan pager \#12345

PCP: William Young

Family Contact: Doris (Wife): 800-555-1111

HPI:

Shortness of breath, found to have pulmonary edema on admission in setting of marked hypertension (BP 200/100), possible non-adherence with anti-hypertensive medications.

Problem List:

Diabetes Mellitus type 2

Chronic Renal Failure (baseline Cr 2.0)

Hypertension

Medications:

Metoprolol $50 \mathrm{mg}$ PO TID

Isordil $40 \mathrm{mg}$ PO TID

Captopril $75 \mathrm{mg}$ PO TID

NPH 40 units SC QAM, 30 units SC at bedtime

Lispro 10 units SC QAC

EcASA $325 \mathrm{mg}$ PO each day

\section{Allergies: NKDA}

Hospital Course:

1. Cardiovascular: Echo showed moderate LVH, EF 60\%. Likely has diastolic dysfunction. 12/28: Blood pressure still hard to control (SBP 120-200). With diuresis and rate control, lungs now clear, $\mathrm{O} 2$ sat $98 \%$ on room air. Titrating blood pressure regimen, renal MRA done today to look for renal artery stenosis, results pending.

2. Diabetes: 12/28: Blood sugars well controlled on current regimen.

3. Renal: $12 / 28$ : Creatinine rose to 3.0 with diuresis, now back to 2.5 .

Tasks:

1. $\square$ Check blood pressure before $10 \mathrm{pm}$. If $\mathrm{SBP}>160$ and pulse $>65$, then increase metoprolol to $100 \mathrm{mg}$ PO TID. If SBP $>160$ and pulse $<65$, then increase isordil to 80 mg PO TID.

2. Renal MRA results may come back tonight, no need to do anything.

Other than labile blood pressure, patient has been stable.

Contingency planning:

1. Do not give benzodiazepines for sleep, patient became delirious earlier in admission when given ativan $1 \mathrm{mg}$ PO. He's written for trazodone qhs prn.

2. Full code.

FIGURE 2. Example of a written sign-out. 
1/5/04. $10: 50 \mathrm{pm}$. Called to see patient for tachycardia noted on monitor. Patient complained of palpitations but denied shortness of breath, chest pain, or lightheadedness. On exam, pulse 140 , blood pressure $125 / 80$, respiratory rate 24 , patient in no apparent distress. Irregular rate and rhythm, no murmur, lungs clear to auscultation. ECG ordered, showed atrial fibrillation at 134, no ST T wave changes compared to previous ECG from 1/4/04. Given previously good response to diltizem, given $10 \mathrm{mg}$ IV with reduction in heart rate to 110. Given $30 \mathrm{mg}$ PO diltiazem.

12 am: no palpitations, back in normal sinus rhythm at 72, blood pressure 110/60.

Signed by

FIGURE 3. Example of patient event note.

\section{The Vehicle for Sign-Out}

We recommend a computer-assisted vehicle for patient information transfer. Ideally, this would be linked to the hospital information system to ensure accurate and up-to-date information Easy access to the computerized sign-out is essential (eg, using a hospitalwide computer system, shared hard drive service, intranet, or PDA linked to the computer system), and it should be customizable for the varied needs of different services and departments. The system should have templates to standardize the content of sign-out, contain robust backup systems, and be HIPAA compliant (ie, restrict access to required health care personnel). However, the perfect should not be the enemy of the good: systems that do not meet these criteria may still help to protect patients by providing legible, predictable, and accessible information.

\section{Sign-Out Processes}

Verbal communication. Although electronic solutions can facilitate the standardization of written content, face-to-face verbal communication adds additional value. ${ }^{19}$ We recommend that each patient be reviewed separately. Identification of each patient verbally ensures that those engaged in the sign-out are discussing the same patient. Reiterating the major medical problems gives a snapshot of the patient and frames the sign-out. The to-do list, the list of tasks that the crosscover resident needs to complete during cross coverage, should be specific and articulated as if, then statements (eg, if the urine output is less than $1 \mathrm{~L}$, then give $40 \mathrm{mg}$ of IV furosemide). The receiver of sign-out should read back to the person giving the sign-out each item on the to-do list (eg, "So, I should check the ins and outs at about 10:00 pm, and give 40 of furosemide if the patient is not $1 \mathrm{~L}$ negative, right?").

Anticipated problems should also be verbally communicated to promote a dialogue. Points that cannot be adequately transferred in the written signout are particularly important to transmit verbally. Examples include previous code discussions, unusual responses to treatment, and psychosocial and family issues. When delivering verbal sign-out, it is important to consider the a priori knowledge of the recipient. How much knowledge about a patient is already shared between the outgoing and incoming physicians and the level of experience of the physicians may affect the extent to which information needs be transmitted. ${ }^{37}$ For instance, 2 experienced physicians who already have been working to cover the same patient will likely have an abbreviated discussion, in contrast to the lengthier sign-out necessary if the outgoing and incoming physicians are interns, and the incoming intern has no prior knowledge of the patient. Similarly, it is likely the level of detail transmitted will need to be greater during a permanent transfer of patient care (ie, at the end of a resident's rotation) than during a brief, temporary transition (eg, overnight coverage).

The challenges of a busy inpatient service may preclude a complete verbal sign-out for all patients; we contend, though, it is best to use these practices to the extent possible, especially for patients with treatment plans in flux, those whose status is tenuous, and those who have anticipated changes in status during cross-coverage. One tool that may be effectively used in signing out such patients is the SBAR tool, according to which a brief description of the situation is given, followed by the background and the physician's specific assessment and complete recommendation. ${ }^{38}$ 
TABLE 2

Checklist for Verbal Communication During Sign-0ut: The Who, What, Where, When, and How

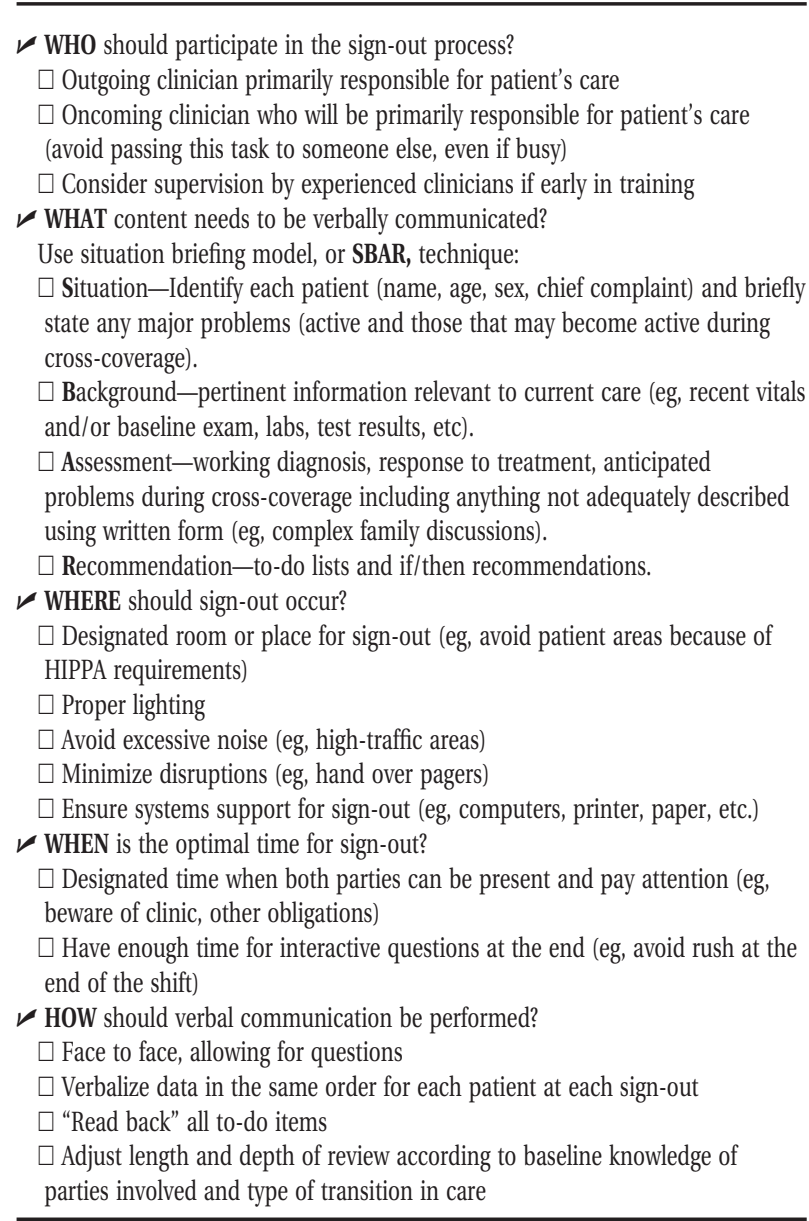

For example, a resident signing out might begin by stating, "I have 18 patients to sign-out to you. I'm going to describe 6 active patients in detail. Twelve others are fairly stable, and I will give you basic information about them, and the details are in the written sign-out. One patient has a plan in flux. The situation is Mr. S. is having trouble breathing, the background is that he has both CHF and COPD, my assessment is that this is more cardiac than pulmonary, and I recommend that you see him first and discuss with the cardiology consultant." Using the tools described here (Table 2), a sign-out of 15 patients of variable acuity could be verbally signed out in less than 10 minutes.

The Environment and setting. To improve the setting of sign-out, we recommend: a designated space that is well lit, quiet, and respects patient confidentiality and a designated time when sign-out will occur. To limit known distractions and interruptions ${ }^{39,40}$ in the hospital, we also recommend the outgoing physician hand off his or her pager to someone else during sign-out. Also key to an environment conducive to information transfer is ensuring adequate computer support for electronic sign-out and access to updated clinical information.

Organizational culture and institutional leadership. The way residents transfer patient care information reflects the culture of the institution. Changing the culture to one in which interactive questioning is valued regardless of position in the hierarchy has been shown to reduce errors in aviation. ${ }^{41}$ Educating residents on the impact of sign-outs on patient care is a first step toward improving the culture of sign-out. Resident commitment to the new sign-out can be gained by engaging residents in development of the process itself. To cement these changes into the culture, practitioners at all levels should be aware of and support the new system. The role of an institution's leaders in achieving these changes cannot be overlooked. Leaders will need to be creative in order to support sign-out as described within the obvious constraints of money, time, personnel, and space. Gaining institutional buy-in can start with heightening the awareness of leaders of the issues surrounding signout, including patient safety, resident efficiency, and the financial impact of discontinuity. Ongoing evaluation of efforts to improve sign-out is also crucial and can be accomplished with surveys, focus groups, and direct observation. Feeding back the positive impact of the changes to all involved stakeholders will promote confidence in the new systems and pride in their efforts.

\section{CONCLUSIONS}

Sign-outs are a part of the current landscape of academic medical centers as well as hospitals at large. Interns, residents, and consulting fellows, not to mention nurses, physical therapists, and nutritionists, transfer patient care information at each transition point. There are few resources that can assist these caregivers in identifying and implementing the most effective ways to transfer patient care information. Hospitals and other care facilities are now mandated to develop standards and systems to improve sign-out. On the basis of the limited literature to date and our own experiences, we have proposed standards and best practices to assist hospitals, training programs, and institutional leaders in designing safe and usable sign-out sys- 
tems. Effective implementation of the standards must include appropriate allocation of resources, individualization to meet specific needs of each program or institution, intensive training, and ongoing evaluation. Future research should focus on developing valid surrogate measures of continuity of care, conducting rigorous trials to determine the elements of sign-out that lead to the best patient outcomes, and studying the most effective ways of implementing these improvements. By improving the content and process of sign-out, we can meet the challenges of the new health care landscape while putting patient safety at the forefront.

Address for correspondence and reprint requests: Arpana R. Vidyarthi, MD, 533 Parnassus Avenue, Box 0131, San Francisco, CA 94143-0131; Fax: (415) 514-2094; E-mail: arpana@medicine.ucsf.edu

Received 24 February 2006; revision received 21 April 2006; accepted 25 April 2006.

\section{REFERENCES}

1. Philibert I, Friedmann P, Williams WT. New requirements for resident duty hours. JAMA. 2002;288:1112-1114.

2. Barden CB, Specht MC, McCarter MD, Daly JM, Fahey TJ 3rd. Effects of limited work hours on surgical training. J Am Coll Surg. 2002;195:531-538.

3. Lockley SW, Cronin JW, Evans EE, et al. Effect of reducing interns' weekly work hours on sleep and attentional failures. N Engl J Med. 2004;351:1829-1837.

4. Landrigan CP, Rothschild JM, Cronin JW, et al. Effect of reducing interns' work hours on serious medical errors in intensive care units. $N$ Engl J Med. 2004;351:1838-1848.

5. Mukherjee S. A precarious exchange. N Engl J Med. 2004; 351:1822-1824.

6. Drazen JM. Awake and informed. N Engl J Med. 2004;351: 1884.

7. Vidyarthi A. Fumbled handoff: missed communication between teams. Cases and Commentary: Hospital Medicine, Morbidity \& Mortality Rounds on the Web. Agency for Healthcare Research and Quality, 2004. Available at: www.webmm.ahrq.gov/case.aspx?caseID $=55$.

8. Healthcare Cost and Utilization Project. Agency for Healthcare Quality and Research. Available at: http://hcup.ahrq.gov/HCUPNet.asp. Accessed May 2004.

9. National Patient Safety Goals. Available at: www.JCAHO.com. Accessed December 2005.

10. Laine C, Goldman L, Soukup JR, Hayes JG. The impact of a regulation restricting medical house staff working hours on the quality of patient care. JAMA. 1993;269:374-378.

11. Petersen LA, Brennan TA, O’Neil AC, Cook EF, Lee TH. Does housestaff discontinuity of care increase the risk for preventable adverse events? Ann Intern Med. 1994;121:866-872.

12. Lofgren RP, Gottlieb D, Williams RA, Rich EC. Post-call transfer of resident responsibility: its effect on patient care. J Gen Intern Med. 1990;5:501-505.

13. Gottlieb DJ, Parenti CM, Peterson CA, Lofgren RP. Effect of a change in house staff work schedule on resource utilization and patient care. Arch Intern Med. 1991;151:2065-2070.
14. Wachter RM, Shojania KG. Internal Bleeding: the Truth behind America's Terrifying Epidemic of Medical Mistakes. New York City: Rugged Land, LLC; 2004:448.

15. Pizzi L, Goldfarb NI, Nash DB. Crew resource management and its applications in medicine. In: Making Health Care Safer: A Critical Analysis of Patient Safety Practices. Evidence Report/Technology Assessment Number 43, AHRQ Publication 01-E058. Rockville, MD: Agency for Healthcare Research and Quality; 2001.

16. Helmreich RL, Klineet JR, Wilhelm JA. System safety and threat and error management: the line operations safety audit (LOSA). In: Jensen RS, ed. Proceedings of the Eleventh International Symposium on Aviation Psychology. Columbus, OH: Ohio State University; 2001:1-6.

17. Thomas EJ, Sexton JB, Helmreich RL. Translating teamwork behaviours from aviation to healthcare: development of behavioural markers for neonatal resuscitation. Qual Saf Health Care. 2004;13(Suppl 1):i57-i64.

18. Patterson ES RB, Woods DD, Chow R, Gomoes JO. Handoff strategies in settings with high consequences for failure: lessons for healthcare operations. Intl J Qual Health Care. 2004;16:125-132.

19. Ambler S. Available at: http://www.agilemodeling.com/essays/communication.htm. Accessed December 15, 2005.

20. Miller C. Ensuring continuing care: styles and efficiency of the handover process. Aust J Adv Nurs. 1998;16:23-27.

21. Manias E, Street A. The handover: uncovering the hidden practices of nurses. Intensive Crit Care Nurs. 2000;16:373383.

22. Sherlock C. The patient handover: a study of its form, function and efficiency. Nurs Stand. 1995;9(52):33-36.

23. Volpp KGM, Grande D. Residents' suggestions for reducing errors in teaching hospitals. N Engl J Med. 2003;348:851-855.

24. Vidyarthi A, Auerbach A. Is 80 the cost of saving lives? Reduced duty hours, errors, and cost. J Gen Intern Med. 2005;20:969-970.

25. Solet DJ, Norvell JM, Rutan GH, Frankel RM. Lost in translation: challenges and opportunities in physician-to-physician communication during patient handoffs. Acad Med. 2005;80:1094-1099.

26. British Medical Association. Safe Handover: Safe Patients: Guidance on Clinical Handover for Clinicians and Managers. London: British Medical Association, Junior Doctors Committee; 2004.

27. Petersen LA, Orav EJ, Teich JM, O’Neil AC, Brennan TA. Using a computerized sign-out program to improve continuity of inpatient care and prevent adverse events. Jt Comm J Qual Improv. 1998;24(2):77-87.

28. Van Eaton EG, Horvath KD, Lober WB, Pellegrini CA. Organizing the transfer of patient care information: the development of a computerized resident sign-out system. Surgery. 2004;136:5-13.

29. Van Eaton EG, Horvath KD, Lober WB, Rossini AJ, Pellegrini CA. A randomized, controlled trial evaluating the impact of a computerized rounding and sign-out system on continuity of care and resident work hours. J Am Coll Surg. 2005;200: 538-545.

30. Ash JS, Gorman PN, Seshadri V, Hersh WR. Computerized physician order entry in U.S. hospitals: results of a 2002 survey. J Am Med Inform Assoc. 2004;11:95-99. 
31. Martin K, Carter L, Balciunas D, Sotoudeh F, Moore D, Westerfield J. The impact of verbal communication on physician prescribing patterns in hospitalized patients with diabetes. Diabetes Educ. 2003;29:827-836.

32. Kilpatrick ES, Holding S. Use of computer terminals on wards to access emergency test results: a retrospective audit. Br Med J. 2001;322:1101-1103.

33. Barenfanger J, Sautter RL, Lang DL, Collins SM, Hacek DM, Peterson LR. Improving patient safety by repeating (readback) telephone reports of critical information. Am J Clin Pathol. 2004;121:801-803.

34. Leonard M GS, Bonacum D. The human factor: the critical importance of effective teamwork and communication in providing safe care. Qual Saf Health Care. 2004;13(Suppl 1):i85-i90.

35. Arora V, Johnson J, Lovinger D, Humphrey HJ, Meltzer DO. Communication failures in patient sign-out and suggestions for improvement: a critical incident analysis. Qual Saf Health Care. 2005;14:401-407.

36. Carty M, Smith C, Schnipper JL. Intern curriculum: the impact of a focused training program on the process and content of signout out patients. Harvard Medical School Education Day, Boston, MA; 2004.

37. Coiera E. When conversation is better than computation. J Am Med Inform Assoc. 2000;7:277-286.

38. SBAR technique for communication: a situational briefing model. Available at: http://www.ihi.org/IHI/Topics/PatientSafety/SafetyGeneral/Tools/SBARTechniqueforCommunicationASituationalBriefingModel.htm. Accessed December 2005.

39. Vidyarthi AKP, Katz PP, Wall SD, Wachter RM, Auerbach AD. Impact of reduced duty hours on residents' educational satisfaction at the University of California, San Francisco. Acad Med. 2006;81:76-81.

40. Coiera E, Tombs V. Communication behaviours in a hospital setting: an observational study. Br Med J. 1998;316:673676.

41. Sutcliffe KM, Lewton E, Rosenthal MM. Communication failures: an insidious contributor to medical mishaps Acad Med. 2004;79(2):186-194. 